\title{
Interview with Bruce Sagan
}

Toufik Mansour

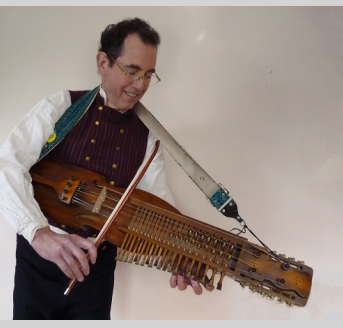

Bruce Sagan obtained his Ph.D. from the Massachusetts Institute of Technology (MIT) in 1979 under the direction of Richard P. Stanley. He is currently a professor of mathematics at Michigan State University. Professor Sagan held visiting positions at different universities: the University of Pennsylvania, Université du Québec, University of California San Diego, Royal Institute of Technology in Stockholm, Newton Institute in England, Mittag-Leffler Institute in Sweden, and the Center for Discrete Mathematics and Theoretical Computer Science at Rutgers University. Professor Sagan has given over 250 lectures, including keynote addresses at the 2006 Formal Power Series and Algebraic Combinatorics Conference in San Diego, the 2011 British Combinatorial Conference in Exeter, and the 2015 Permutations in Patterns Conference in London. His research interests include algebraic, enumerative, and topological combinatorics as well as graph theory. In particular, he works on symmetric functions, groups and representations, tableaux algorithms, chromatic polynomials of graphs, the Möbius function and homology of partially ordered sets, pattern avoidance and containment in permutations and other combinatorial structures. Since 2004, professor Sagan has been an Editor-in-Chief of the Electronic Journal of Combinatorics.

Mansour: Professor Sagan, first of all, we would like to thank you for accepting this interview. Would you tell us broadly what combinatorics is?

Sagan: I think of combinatorics as the study of discrete structures. It can be broadly broken down into three major subdisciplines. There is the enumerative side where I work, with the emphasis being on counting structures. Another major area is extremal combinatorics where the focus is on finding a structure which is "best" according to some criteria. Finally, there is the existential realm where one asks if a structure with certain properties even exists. The field is so large now that it can be hard for researchers in one area to keep track of what is going on in the others. It was for this reason that during my time as a Program Officer at NSF I insisted that rather than having one panel for all of combinatorics, we have two. velopment of the relations between combinatorics and the rest of mathematics?

Sagan: Before the middle of the last century, combinatorics was considered secondrate mathematics, just a bag of tricks. This all changed when Gian-Carlo Rota started to show the mathematical world that the field had substance through his sequence of articles "On the foundations of combinatorial theory." Today it is generally accepted that combinatorics is an important area of research. This has been helped by the fact that many modern combinatorialists use techniques from other areas such as algebra, topology, and analysis in their work. And the cross-fertilization goes the other way with people using combinatorics to solve problems of interest to people outside the field.

Mansour: What have been some of the main goals of your research?

Mansour: What do you think about the de- Sagan: I am very excited by the interactions

The authors: Released under the CC BY-ND license (International 4.0), Published: January 22, 2021

Toufik Mansour is a professor of mathematics at the University of Haifa, Israel. His email address is tmansour@univ.haifa.ac.il 
between combinatorics and other fields. In particular, I am fascinated by problems that involve ideas from algebra or topology. An example of the former is Stanley's generalization of the chromatic polynomial to symmetric functions which is one of my favorite areas of research. In term of the latter, the fact that the Möbus function of a partially ordered set can be viewed as an Euler characteristic is a wonderful result. Much of my work concerns properties of Möbus functions.

Mansour: We would like to ask you about your formative years. What were your early experiences with mathematics? Did that happen under the influence of your family or some other people?

Sagan: I was interested in mathematics from a young age. I remember when I was shown the algorithm for taking square roots in grade school working out the analogue for arbitrary roots. Similarly, in high school my trigonometry class covered the double-angle formulas for sine and cosine which set me to finding a formula for any integral multiple of an angle, thus rediscovering the Chebyshev polynomials.

Science seems to run in the Sagan family as witness my cousin Carl Sagan (the famous astronomer), and my brother David Sagan who works in high-energy physics at Cornell. Perhaps the person who had the most influence over me was a fellow name Dr. Weltin. He ran after school classes at the Berkeley Lawrence Hall of Science for bright high school students teaching them calculus. It was not so common then to be introduced to calculus before college.

Mansour: Were there specific problems that made you first interested in combinatorics?

Sagan: The reason I became interested in the field was because I took a graduate combinatorics class from Richard Stanley during my first year at MIT. For a final project, we had to read a paper in the literature and report on it. I chose one about chromatic polynomials. After finishing the paper, I decided to try and compute the polynomial for a graph not considered in the article. I did this for the complete bipartite graph and included it in my report. So it seemed as if I had some aptitude for this material and decided to work in the area.

Mansour: What was the reason you chose MIT for your Ph.D. and your advisor Richard Stanley?

Sagan: MIT was a difficult choice for me. I did my undergraduate work at Cal State Hayward (now Cal State East Bay). I got excellent letters of recommendation from my teachers, but because of the obscurity of the school it must have been hard for the places where I applied to judge my abilities. I got into graduate school at both MIT and the University of Michigan. I preferred MIT academically, but they were not willing to give me any money while Michigan was offering a full scholarship. Finally I decided on MIT and have never regretted the choice. And MIT did support me after the first year. I think they just waited to see how I was doing in competition with their own students. As far as choosing Richard as my advisor, I think that should be obvious given my answer to the previous question.

Mansour: What was the problem you worked on in your thesis?

Sagan: I worked on finding analogues for shifted Young tableaux of various algorithms for left-justified tableaux. In particular, I came up with a shifted version of the RobinsonSchensted (RS) correspondence ${ }^{1,2,3}$. But it did not have any of the nice properties of the original RS algorithm. Several years later while I was a postdoc in Wales, I came up with a much nicer bijection. I was very excited and wrote a letter to Richard outlining it's various properties. A few days after I put it in the mail (this was before the age of computers) and so long before Richard would have received my missive, I got a letter from him. He wrote that he had a graduate student, Dale Worley, who had come up with a better shifted RS algorithm which had the following properties. His list was almost identical to mine! Thus was born the Sagan-Worley correspondence ${ }^{4,5}$.

Mansour: What would guide you in your research? A general theoretical question or a specific problem?

\footnotetext{
${ }^{1}$ C. Schensted, Longest increasing and decreasing subsequences, Canad. J. Math. 13 (1961) $179-191$.

${ }^{2} \mathrm{G}$. de B. Robinson, On the representations of the symmetric group, Amer. J. Math. 60(3) (1938) $745-760$.

${ }^{3}$ B.E. Sagan, An analog of Schensted's algorithm for shifted Young tableaux, J. Combin. Theory Ser. A 27 (1979), $10-18$.

${ }^{4}$ B.E. Sagan, Shifted tableaux, Schur Q-functions, and a conjecture of R. P. Stanley, J. Combin. Theory Ser. A 45 (1987), $62-103$.

${ }^{5}$ D. Worley, A Theory of Shifted Young Tableaux, Ph.D. thesis, MIT, 1984.
} 
Sagan: I do research based on both theory and specific problems, sometimes mixing the two up on a given topic. A good example of this comes from some work I am currently doing. It started out when I attended a lecture by Valentin Ovsienko where he presented, among other things, a lovely conjecture he had made with Sophie Morier-Genoud ${ }^{6}$ about the rank unimodality of certain distribute lattices arising from posets called fences. I had some ideas about how to prove various special cases of the conjecture and this has blossomed into a preprint on the arXiv with Thomas McConville and Clifford Smyth ${ }^{7}$. Shortly after we finished the paper, I was invited to a conference on dynamical algebraic combinatorics, an area I knew very little about but which I was interested in investigating. After reading some introductory material, I realized that fences might have some very pretty associated dynamics. This has now become a joint research project with various other people who attended the conference.

Mansour: When you are working on a problem, do you feel that something is true even before you have the proof?

Sagan: Certainly when I am working on a conjecture I am hoping it is true. But this can also be a trap, since if the conjecture is false then I may be wasting my time. A good piece of advice which was given to me (unfortunately, I ca not remember by whom) is that when working on such a question one should alternate: one day trying to prove it is true and the next attempting to show it is false.

Mansour: What three results do you consider the most influential in combinatorics during the last thirty years?

Sagan: One of the most influential series of results in extremal graph theory has been the Graph Minors Project of Neil Robinson and Paul Seymour ${ }^{8}$. They clearly showed that
Wagner's Theorem ${ }^{9}$ (a graph is planar if and only if it has no minor isomorphic to $K_{5}$ or $\left.K_{3,3}\right)$ is the proverbial tip of the iceberg. Their many deep theorems on the subject include showing that the minor relation is a well-quasiordering and their Graph Structure Theorem determining, for any given graph $G$, the structure of any graph not having $G$ as a minor.

Another incredibly fruitful viewpoint was the invention of cluster algebras by Sergey Fomin and Andrei Zelevinsky ${ }^{10,11}$. These objects have broad application in combinatorics, geometry, and (of course) algebra. In fact, there has been such an explosion of work on this topic that Fomin maintains a cluster algebra portal with links to papers, conferences, and other related material.

Finally, and most closely related to my work, there was June Huh's ${ }^{12}$ spectacular proof in 2013 of the conjecture that the coefficients of the chromatic polynomial form a log concave sequence. The proof used deep results from algebraic geometry such as Chern classes. Since then, Huh and his collaborators have gone on to formulate a number of new methods for obtaining results about various families of polynomials.

Mansour: What are the top three open questions in your list?

Sagan: There are three questions related to my work that I would dearly love to have answered. The one I have worked on the longest is the $(\mathbf{3}+\mathbf{1})$-free Conjecture of Richard Stanley and John Stembridge ${ }^{13}$. Stanley ${ }^{14}$ defined a symmetric function $X(G)$ associated with any finite graph $G$ which reduces to the chromatic polynomial under specialization of the variables. It is then natural to ask what can be said about $X(G)$ when expanded in the various standard bases for the algebra of symmetric functions. Associated with any finite poset $P$ is a graph $G_{P}$ whose vertices are the ele-

\footnotetext{
${ }^{6}$ S. Morier-Genoud and V. Ovsienko, q-deformed rationals and q-continued fractions, Forum Math. Sigma 8 (2020) e13.

${ }^{7}$ T. McConville, B.E. Sagan and C. Smyth, On a rank-unimodality conjecture of Morier-Genoud and Ovsienko, arXiv:2008.13232, 2020 .

${ }^{8}$ N. Robertson and P. Seymour, Graph minors. XVIII. Tree-decompositions and well-quasi-ordering, J. Combin. Theory Ser. B 89 (2003) 77-108.

${ }^{9}$ K. Wagner, Über eine eigenschaft der ebenen komplexe, Math. Ann. 114 (1937) 570-590.

${ }^{10}$ S. Fomin and A. Zelevinsky, Cluster algebras. I. Foundations, J. Amer. Math. Soc. 15:2 (2002) 497-529.

${ }^{11}$ S. Fomin, and A. Zelevinsky, Cluster algebras. II. Finite type classification, Invent. Math. 154:1 (2003) 63-121.

$12 \mathrm{~J}$. Huh, Milnor numbers of projective hypersurfaces and the chromatic polynomial of graphs, J. Amer. Math. Soc. 25(3) (2012) 907-927.

${ }^{13}$ R.P. Stanley and J. Stembridge, On immanants of Jacobi-Trudi matrices and permutations with restricted position, J. Combin. Theory Ser. A 62 (1993), 261-279.

${ }^{14}$ R.P. Stanley, A symmetric function generalization of the chromatic polynomial of a graph, Advances in Math. 111 (1995), $166-194$.
} 
ments of $P$ with an edge connecting $x$ and $y$ if these elements are incomparable in $P$. Say that $P$ is $(\mathbf{3}+\mathbf{1})$-free if it contains no induced subposet isomorphic to a disjoint union of a 3element chain and a 1-element chain. The conjecture states that, for a $(\mathbf{3}+\mathbf{1})$-free poset $P$, if $X\left(G_{P}\right)$ is expanded in the elementary symmetric function basis then all of the coefficients are nonnegative. Vesselin Gasharov ${ }^{15}$ proved the weaker statement that the expansion in the Schur basis has nonnegative coefficients. And recently Mathieu Guay-Paquet ${ }^{16}$ showed that it suffices to consider posets which are both $(\mathbf{3}+\mathbf{1})$ - and $(\mathbf{2}+\mathbf{2})$-free. With my then graduate student ${ }^{17}$, David Gebhard, we were able to verify some special cases of this conjecture using the symmetric functions in noncommuting variables which I introduced with Mercedes Rosas $^{18}$. This work has been continued by Samantha Dahlberg and Stephanie van Willigenburg $^{19}$

Another conjecture which I have become interested in more recently is the one I described above by Morier-Genoud and Ovsienko ${ }^{20}$. The frustrating thing is that we have a method which we believe (and which we have tested by computer on many examples) will generate nested chain decompositions of these lattices. This would immediately imply rank unimodality as well as the strong Sperner property. But we have been unable to prove that this algorithm always works.

Finally, even though we now know from Huh's work that the coefficients of the chromatic polynomial are log concave, it would be wonderful to have a combinatorial proof. For example, maybe there is some way to use the powerful directed path method of Bernt Lindström $^{21}$, Ira Gessel, and Gérard (now Xavier) Viennot $^{22}$.

Mansour: What kind of mathematics would you like to see in the next ten-to-twenty years as the continuation of your work?

Sagan: I hope that combinatorics will con- tinue its current trend of interacting with other fields of mathematics. I think this is very important for the vitality of the subject.

Mansour: Do you think that there are core or mainstream areas in mathematics? Are some topics more important than others?

Sagan: I am very wary of assigning importance to different types of mathematics. As mentioned above, at one point combinatorics was considered a backwater of mathematics. But this is definitely not the prevailing opinion now.

Mansour: What do you think about the distinction between pure and applied mathematics that some people focus on? Is it meaningful at all in your case? How do you see the relationship between so-called "pure" and "applied" mathematics?

Sagan: Just as with my answer to the previous question, I don't think focusing on whether something is pure or applied is a profitable way of assessing the worth of a piece of research. And ideas which started out as purely theoretical have latter been shown to have applications. An example of this is the RSA algorithm in cryptography which is based on ideas from number theory.

Mansour: What advice would you give to young people thinking about pursuing a research career in mathematics?

Sagan: I think we need to be careful to tell them both the positive and negative aspects of pursing such a career. On the positive side, if you have a mathematics degree there are many opportunities for working in industry. If the person is thinking about an academic job, it is important to realize that these positions have become much harder to get. But if one manages to obtain a tenure-track position, then the life of a professor has many advantages, including being able to choose the problems you want to work on rather than having them dictated by the company for which you work.

Mansour: Would you tell us about your interests besides mathematics?

\footnotetext{
${ }^{15} \mathrm{~V}$. Gasharov, Incomparability graphs of $(\mathbf{3}+\mathbf{1})$-free posets are s-positive, Discrete Math. 157 (1996) $211-215$.

${ }^{16} \mathrm{M}$. Guay-Paquet, A modular relation for the chromatic symmetric functions of (3+1)-free posets, (2013) arXiv:1306.2400.

${ }^{17}$ D.D. Gebhard and B.E. Sagan, A chromatic symmetric function in noncommuting variables, J. Algebraic Combin. 13 (2001) $227-255$.

${ }^{18}$ M. Rosas and B. Sagan, Symmetric functions in noncommuting variables, Trans. Amer. Math. Soc. 358 (2006), $183-214$.

${ }^{19}$ S. Dahlberg and S. van Willigenburg, Chromatic symmetric functions in noncommuting variables revisited, Adv. in Appl. Math. 112 (2020) 101942.

${ }^{20}$ T. McConville, B.E. Sagan and C. Smyth, On a rank-unimodality conjecture of Morier-Genoud and Ovsienko, (2020) arXiv:2008.13232.

${ }^{21} \mathrm{~B}$. Lindström, On the vector representations of induced matroids, Bull. London Math. Soc. 5 (1973), 85-90.

${ }^{22}$ I. Gessel and G. Viennot, Binomial determinants, paths, and hook length formulae, Adv. in Math. 58 (1985), $300-321$.
} 
Sagan: I am very interested in languages and in addition to English I can speak Swedish and French fluently. I have also dabbled in Spanish, German and Bulgarian. I do a lot of traveling and I think it is polite to at least learn the basic pleasantries if one is visiting a country.

Mansour: We see that you have a strong passion for music and you even produced four albums. From your music-related web page ${ }^{23}$, we learn that you play several Balkan and Scandinavian instruments (gûdulka, hardingfele, nyckelharpa, and fiddle). How did you become interested in music and specifically Balkan music? Which instruments can you play at the professional level?

Sagan: My mother was a music teacher and later a choir director, so I studied classical violin from an early age. I became interested in international folk dancing when I was in college. And dancers became my main community of friends. So when I came to MIT, one of the first things I did was find the folk dance group on campus. During that time, people usually danced to recorded music. But one evening a live band came to play. I had already had it in the back of my mind to try and learn the music to which I was dancing, and so I came up to the musicians afterwards and asked if they wanted somebody to play Hungarian violin. Now, I had never played Hungarian folk music outside of some compositions of Bartók. But I figured I was classically trained while these people probably could not even read music. As you can see, I had my nose firmly up in the air. They invited me to their next rehearsal and put the sheet music for a Bulgarian rûchenitsa in front of me. This is a dance in $7 / 16$, a meter I had never played before, and this particular tune was from the Shope region where they dance like the wind. The band took off playing leaving me firmly in their dust. So I learned that these musicians had something to teach me! Later I found out that they had been working on the piece all summer and I was the only one who was sight reading. But still, it was a valuable lesson. Interestingly, a number of Stanley's students are interested in folk dance and music including Ira Gessel and Tom Roby.

Later I started specializing and gravitated towards the music of Scandinavia, where the fiddle (which is the same as a violin) is king, and the music of Bulgaria because of its odd time signatures. I am now what I would call a semi-professional musician. I am paid for playing, but I do not take enough gigs to support myself. I have recorded $4 \mathrm{CDs}$ and founded a camp for Scandinavian Music and Dance which is still running today after 35 years even though I have stepped down as music director. Mansour: You helped develop a computer package for extracting the backbone from a bipartite graph. What is a backbone?

Sagan: Thus is a project ${ }^{24}$, I am working on with my graduate student, Rachel Domagalski, and my colleague in the Psychology Department here at MSU, Zachary Neal. Suppose we have a set which we call the agents and we want to study interactions between them. For example, the agents could be the members of the US Senate and we want to study which senators work together. This information may be hard to observe directly, so we consider another set whose elements are called artifacts. This gives us a bipartite graph where the two partite sets are the agents and the artifacts and there is an edge if an agent is associated with an artifact. Returning to our example, we could use bills considered by the Senate as artifacts and connect a senator and a bill if the senator sponsors that bill. From this graph we get a weighted graph, called the projection, whose vertices are the agents and the weight of an edge between two agents is the number of artifacts they share. But this graph may be very dense and hard to interpret. So one would like to find a backbone for it consisting of only the edges which are most significant. There are a number of different algorithms which have been proposed for extracting a backbone, but nobody had previously made them available on the same software platform, tested the various algorithms for speed and how well their backbones corresponded to the suspected underlying ground truth, etc. That was the purpose of the package we developed which has now been downloaded over 10,000 times. Together with a larger team of researchers from MSU, we are now working on a more general project for extracting the backbone of an arbitrary weighted

\footnotetext{
${ }^{23}$ https://www.brucesagan.com/

${ }^{24}$ R. Domagalski, Z. Neal and B.E. Sagan, backbone: An R Package for extracting the backbone of bipartite projections, PLoS One, (2021), 20 pp. (electronic).
} 
graph which may not have come from a bipartite projection.

Mansour: Your book The Symmetric Group: Representations, Combinatorial Algorithms, and Symmetric Functions ${ }^{25}$ has become a key reference on combinatorics and representation theory of symmetric group. The research at the intersection of combinatorics and representation theory is growing very fast. Do you have any plan to write another book on this topic?

Sagan: I am not planning another book on this topic. But I have recently written a new book about enumerative combinatorics. It is called Combinatorics: The Art of Counting and has just been published by the AMS as part of the Graduate Studies in Mathematics Series $^{26}$

It is an introduction to enumeration at the advanced undergraduate or beginning graduate level, similar to that of my symmetric group book. I cover both standard material (e.g., generating functions, posets, group actions, symmetric and quasisymmetric functions) as well as some topics which have never appeared in textbook form before (e.g., the theory of quotient posets which I developed with my former student Joshua Hallam ${ }^{27}$ ). I have tried hard to make the presentation user friendly and suitable for self study.

Mansour: One of your research interests is about pattern avoidance and containment in permutations. The study of permutation patterns has been a focus of many researchers in the last thirty years and we have seen great advances. What did you think about this research overall and recent progresses? What kind of questions do you think will lead to new research directions for the study of permutation patterns?

Sagan: The permutation patterns community started out as rather small and inward looking, motivated mainly by enumeration questions and the Stanley-Wilf Conjecture which was later proved by Adam Marcus and Gábor Tar$\operatorname{dos}^{28}$. More recently, the field has expanded greatly both in terms of the number of people doing research and in the types of questions be- ing asked. Connections are being forged with probabilistic combinatorics (random permutations, distributions of permutations), algebraic combinatorics (computation of Möbius functions, connections with quasisymmetric functions), algebraic geometry (characterization of smooth and other types of varieties), and so forth. I think this is very healthy and feel that these are the directions people should continue to pursue. For example, the question of completely determining the Möbius function for intervals in the poset of permutations ordered by pattern containment is far from being resolved. And of course one could ask for even more refined information such as the homological structure of these intervals.

Mansour: In your 2012 paper, Permutation patterns and statistics ${ }^{29}$, you introduced a natural generalization of the theory of Wilfequivalence of permutations by using permutation statistics. Would you tell us about recent developments on this idea and point out some future research directions?

Sagan: The most recent development I know of is current research I am doing with some students at MSU: undergraduates Jamie Schmidt and Alex Sietsema and my Ph.D. students Rachel Domagalski, Jinting Liang, and Quinn Minnich. There has been recent interest it circular analogues of various combinatorial objects. In particular, one can define pattern containment for cyclic permutations as one does for linear ones, just allowing the pattern to wrap around from the end of a permutation to the beginning. In a recent paper of David Callan ${ }^{30}$, he gives the cardinalities of all the classes of circular permutations avoiding a single pattern of length four. In our work, we consider all possible sets of patterns of length four and determine the generating functions for their avoidance classes with respect to the number of circular descents and circular peaks. Several nontrivial generalized Wilf-equivalences result.

As far as future directions, one could generalize Wilf-equivalence even further from nonnegative integer-valued statistics to any per-

\footnotetext{
${ }^{25}$ See, https://www.springer.com/gp/book/9780387950679

${ }^{26}$ See, https://bookstore.ams.org/gsm-210/

${ }^{27}$ J. Hallam and B. Sagan, Factoring the characteristic polynomial of a lattice, J. Combin. Theory Ser. A, 136 (2015), $39-63$.

${ }^{28}$ A. Marcus and G. Tardos, Excluded permutation matrices and the Stanley-Wilf conjecture, J. Combin. Theory Ser. A 107(1) (2004) 153-160.

${ }^{29}$ T. Dokos, T. Dwyer, B.P. Johnson and K. Selsor, Permutation patterns and statistics, Discrete Math., 312 (2012), $2760-2775$.

${ }^{30}$ D. Callan, Pattern avoidance in circular permutations, (2002) arXiv:math/0210014v1
} 
mutation statistic by considering two sets of patterns equivalent if there is a bijection between their avoidance classes which preserves both length and the statistic. It would be interesting to study this idea. For example, in a recent paper with Zachary Hamaker and Brendan Powlowski ${ }^{31}$, we associate with any avoidance class a quasisymmetric function and then compute this function for certain sets of patterns having length three. (This work was continued in an article I wrote with Jonathan Bloom $^{32}$.) Some of these results can be interpreted as giving Wilf equivalences for the descent set statistic.

Mansour: In one of your works, co-authored with Andreas Blass Möbius functions of lattices $^{33}$, you introduced the concept of a bounded below set in a lattice. This was used to generalize Gian-Carlo Rota's ${ }^{34}$ broken circuit theorem to any finite lattice. Would you tell us briefly about the context and motivation for this concept?

Sagan: Rota generalized Whitney's ${ }^{35}$ characterization of the coefficients of the chromatic polynomial of a graph in terms of broken circuits to determining the Möbius function of any geometric lattice in terms of NBC (no broken circuit) sets. It is natural to ask if one could generalize even further and find an analogous formula for an arbitrary lattice, geometric or not. This is what I did with Blass.

Mansour: You have numerous papers on symmetric functions. Why is it interesting to study them? Can you give us some examples where they arise naturally? Do they also appear in some context in an unexpected way?

Sagan: Symmetric functions are interesting because they connect various fields of mathematics. They are of interest to algebraists because they can be viewed as the generating functions for the irreducible characters of the symmetric group, or the polynomial characters of the general linear group. They are of use in combinatorics for many reasons. For example, the Schur symmetric functions are the generating functions for semistandard Young tableaux and play a fundamental role in the RobinsonSchensted-Knuth ${ }^{36}$ correspondence. Also, the power sum symmetric functions appear naturally in the Redfield ${ }^{37}$-Pólya ${ }^{38}$ theory of enumeration under group action. As far as an unexpected occurrence, Stanley's definition of the chromatic symmetric function discussed previously was a dramatic new idea. It has since proved to be very fruitful.

Mansour: The title of one of your very recent papers is Lucas atoms ${ }^{39}$. How are they defined? How are they related to Lucas polynomials? What about their possible combinatorial interpretation?

Sagan: Let $s, t$ be two variables. The Lu$\operatorname{cas}^{40}$ sequence is a sequence of polynomials in $s$ and $t$, with the $n$th polynomials denoted $\{n\}$, satisfying the initial conditions $\{0\}=0$, $\{1\}=1$, and for $n \geq 2$ we define $\{n\}=$ $s\{n-1\}+t\{n-2\}$. Note that specializing $s=t=1$ one recovers the Fibonacci numbers. Even more interesting, letting $s=1+q$ and $t=-q$ for a new variable $q$ turns $\{n\}$ into the standard $q$-analogue of an element of $\mathbb{N}$, the nonnegative integers. So, just as in the theory of $q$-analogues, we can produce Lucas analogues of various combinatorial constants such as binomial coefficients or Catalan numbers by replacing each factor of $n$ by $\{n\}$ in their usual definition as a quotient of products. One can then ask when the resulting rational function of $s, t$ is actually a polynomial. And in that case when the polynomial will have coefficients in $\mathbb{N}$.

In joint work with Jordan Tirrell based on an idea of Richard Stanley, we define polynomials $P_{d}(s, t) \in \mathbb{N}[s, t]$ which we call Lucas atoms such that $\{n\}=\prod_{d \mid n} P_{d}(s, t)$. These polynomials have the wonderful property that a Lucas analogue will be a polynomial if and

\footnotetext{
${ }^{31}$ Z. Hamaker, B. Pawlowski and B.E. Sagan, Pattern avoidance and quasisymmetric functions, Algebr. Comb. 3:2 (2020) 365-388.

32 J.S. Bloom and B.E. Sagan, Revisiting pattern avoidance and quasisymmetric functions, Ann. Combin. 24 (2020) $337-361$.

${ }^{33}$ A. Blass and B.E. Sagan, Möbius functions of lattices, Adv. Math. 127:1 (1997) 94-123.

${ }^{34}$ G.-C. Rota, On the foundations of combinatorial theory I. Theory of Möbius functions, Z. Wahrscheinlichkeitstheorie 2 (1964), $340-368$.

${ }^{35} \mathrm{H}$. Whitney, A logical expansion in mathematics, Bull. Amer. Math. Soc. 38 (1932), 572-579.

${ }^{36}$ D.E. Knuth, Permutations, matrices and generalized Young tableaux, Pacific J. Math. 34 (1970), $709-727$.

${ }^{37}$ J.H. Redfield, The theory of group-reduced distributions, Amer. J. Math. 49 (1927), 433-455.

${ }^{38}$ G. Pólya, Kombinatorische Anzahlbestimmungen für Gruppen, Graphen und chemische Verbindungen, Acta Math. 68 (1937), $145-254$.

${ }^{39}$ B.E. Bruce and J. Tirrell, Lucas atoms, Adv. Math. 374:18 (2020) 107387.

${ }^{40}$ E. Lucas, Théorie des fonctions numériques simplement périodiques, Am. J. Math. 1 (2) (1878), $184-196$.
} 
only if, after expressing the top and bottom in terms of atoms, all the $P_{d}$ in the denominator cancel into the numerator. It follows immediately that if a Lucas analogue is a polynomial then it must have nonnegative integer coefficients! It turns out that this is a powerful method for proving polynomiality and we do this for Lucas analogues of binomial coefficients as well as Fuss-Catalan and Fuss-Narayana numbers for all irreducible Coxeter groups. Furthermore, it turns out that $P_{n}(1+q,-q)=\Phi_{n}(q)$, the $n$th cyclotomic polynomial. So we also prove $s, t$ version of various properties of the $\Phi_{n}(q)$ such as sum-ofsquares theorems of Gauss ${ }^{41}$ and Lucas ${ }^{42}$, and reduction formulas.

The $\{n\}$ have a very simple combinatorial interpretation in terms of tilings of a strip with tiles covering either one or two squares. We tried very hard to come up with a nice interpretation for the $P_{n}(s, t)$ and failed. I would add this to my list of problems that I would very much like to see solved.

Mansour: In your work, you have extensively used combinatorial reasoning to address important problems. How do enumerative techniques engage in your research?

Sagan: Most of what I do has some enumerative aspect. Even when I employ algebraic or topological techniques, they are usually in the service of some problem of enumeration. I also like problems where one needs to come up with combinatorial interpretations for objects that appear in an algebraic or topological context. Examples include the NBC description of the Möbius function or the desired combinatorial interpretation of the coefficients of the Lucas atoms mentioned previously.

Mansour: Among all combinatorial objects we are unable to enumerate, which one do you wish to see enumerated soon?

Sagan: It is well known that the generating functions for linear and plane partitions have beautiful product expansions. To date, nobody has come up with nice way to enumerate three-dimensional partitions. Of course, it may not be possible to do so. But for a long time nobody knew of a good expression for the number of standard Young tableaux of a given skew shape analogous to the famous Frame-Robinson-Thrall ${ }^{43}$ hook formula for left-justified shapes. But recently Naruse ${ }^{44}$ came up with such an expression as a sum of products, rather than a single product as in the FRT case.

Mansour: Would you tell us about your thought process for the proof of one of your favorite results? How did you become interested in that problem? How long did it take you to figure out a proof? Did you have a "eureka moment"?

Sagan: As I mentioned before, the shifted Robinson-Schensted algorithm which I discovered for my thesis was not as flexible as the left-justified version. I lectured about it at the first conference I ever attended (at Oberwolfach). One of the participants asked me if there might be another way to construct the map which would have more of the desired properties. I answered that it might be possible, but I didn't think so. These were words I came to regret, but they also put the seed in my mind to search for a better bijection. So when I took the postdoc in Wales, it was on my list to revisit this problem. I still remember the night when I couldn't sleep and so went into the kitchen to work. Then in a flash I saw that if I used both row insertion and column insertion (as opposed to just the former as I had done earlier) then I might be able to recover the desired properties.

Mansour: Is there a specific problem you have been working on for many years? What progress have you made?

Sagan: I think the problem I have spent the most time working on is the $(\mathbf{3}+\mathbf{1})$-free Conjecture described above. As mentioned there my main contribution has been the use of symmetric functions in noncommuting variables as a tool.

Mansour: Professor Bruce Sagan, I would like to thank you very much for this very interesting interview on behalf of the journal Enumerative Combinatorics and Applications.

\footnotetext{
${ }^{41}$ C.F. Gauss, Disquisitiones Arithmeticae, Springer-Verlag, New York, 1986. Translated and with a preface by A.A. Clarke, revised by W.C. Waterhouse, C. Greither and A.W. Grootendorst and with a preface by Waterhouse.

${ }^{42}$ H. Riesel, Prime Numbers and Computer Methods for Factorization, second edition, Progress in Mathematics, vol. 126, Birkhäuser Boston, Inc., Boston, MA, 1994.

${ }^{43}$ J.S. Frame, G. de B. Robinson, and R.M. Thrall, The hook graphs of the symmetric groups, Canad. J. Math. 6 (1954) 316-324.

${ }^{44}$ H. Naruse, Schubert calculus and hook formula, Talk slides at 73rd Sém. Lothar. Comin., Strobl, Austria, 2014; available at tinyurl.com/z6paqzu.
} 